\title{
O caramujo exótico invasor Achatina fulica (Stylommatophora, Mollusca) no Estado do Rio de Janeiro (Brasil): situação atual
}

\author{
Joana Zanol ${ }^{1,2,3}$, Monica Ammom Fernandez ${ }^{2}$, Ana Paula Martins de Oliveira ${ }^{2}$, \\ Claudia Augusta de Moraes Russo ${ }^{1}$ \& Silvana Carvalho Thiengo ${ }^{2}$ \\ ${ }^{1}$ Departamento de Genética, Instituto de Biologia, Universidade Federal do Rio de Janeiro, \\ Av. Prof Rodolpho Paulo Rocco, s/n, CCS, Bloco A, A2-097, CEP 21941-617, \\ Ilha do Fundão, Rio de Janeiro, RJ, Brasil, e-mail: claudiaamrusso@gmail.com \\ ${ }^{2}$ Laboratório de Referência Nacional em Malacologia Médica, Fundação Instituto Oswaldo Cruz - Fiocruz, \\ Av. Brasil, 4365, CEP 21040-900, Manguinhos, Rio de Janeiro, RJ, Brasil, e-mail: ammon@ioc.fiocruz.br, \\ apmartin@ioc.fiocruz.br, sthiengo@ioc.fiocruz.br \\ ${ }^{3}$ Autor para correspondência: Joana Zanol,e-mail: jzanol@gwmail.gwu.edu
}

ZANOL, J., FERNANDEZ, M.A., OLIVEIRA, A.P.M. \& THIENGO, S.C., The exotic invasive snail Achatina fulica (Stylommatophora, Mollusca) in the State of Rio de Janeiro (Brazil): current status. Biota Neotrop., 10(3): http://www.biotaneotropica.org.br/v10n3/en/abstract?short-communication+bn00610032010.

\begin{abstract}
The invasive African snail Achatina fulica was introduced in Brazil through South and Southeast States in at least three separate occasions. A. fulica is currently present in 24 out of 26 Brazilian States and in the Federal District. Dense populations of A. fulica are nuisance to human populations and pest to gardens and small crops. Such populations also act in the transmission of two zoonosis (abdominal angiostrongyliasis and eosinophilic meningitis) as well as other parasitosis of veterinary importance. Here, we report new records of A. fulica and of nematode larvae of medical and veterinary importance found in this snail in the state of Rio de Janeiro (Brazil). A. fulica is present in 26 additional municipalities of the state when compared to the information obtained in 2006, year of the last census. This result represents an increase of 50\% in the number of municipalities infested. Only nine out of the 92 municipalities of the state have not yet registered the presence of this invasive species. Nematode larvae of Aelurostrongylus abstrusus, Rhabditis sp. e Strongyluris-like, all of veterinary importance, have been registered in A. fulica individuals from eleven municipalities of the state. The rapid range expansion of $A$. fulica calls attention for the need of a general and continuous management plan throughout the country in order to effectively control the invasion. The current level of infestation makes a full eradication unlikely.

Keywords: Achatina fulica, Gastropoda, invasive species, distribution, public health.
\end{abstract}

ZANOL, J., FERNANDEZ, M.A., OLIVEIRA, A.P.M. \& THIENGO, S.C., O caramujo exótico invasor Achatina fulica (Stylommatophora, Mollusca) no Estado do Rio de Janeiro (Brasil): situação atual. Biota Neotrop., 10(3): http://www.biotaneotropica.org.br/v10n3/pt/abstract?short-communication+bn00610032010.

Resumo: O caramujo africano invasor Achatina fulica foi introduzido no Brasil a partir de Estados do Sul e Sudeste em pelo menos três ocasiões. Atualmente sua distribuição já abrange 24 dos 26 estados e o Distrito Federal. Populações densas dessa espécie vêm causando incômodos à populações humanas, danos à jardins e pequenas plantações, além de atuarem como transmissoras de duas zoonoses (angiostrongilíase abdominal e meningoencefalite eosinofílica) e outras parasitoses de interesse veterinário. No presente estudo, apresentamos novas ocorrências de A. fulica no Estado do Rio de Janeiro (Brasil) assim como dados sobre as larvas de nematódeos de interesse médico e veterinário encontradas em exemplares dessa espécie coletados neste estado. A. fulica está presente em 26 municípios adicionais em relação aos dados obtidos em 2006, ano do último levantamento, representando um aumento de 50\% no número de municípios infestados. Apenas nove dos 92 municípios do estado ainda não registram a presença dessa espécie. Larvas dos nematódeos Aelurostrongylus abstrusus, Rhabditis sp. e Strongyluris-like, todas com importância veterinária, foram encontradas em exemplares de A. fulica de onze municípios. A rápida dispersão de A. fulica evidencia a necessidade de planos de manejo contínuos e eficientes em todo o país visando o controle efetivo dessa invasão. A erradicação de A. fulica é pouco provável devido ao nível atual de infestação.

Palavras-chave: Achatina fulica, Gastropoda, espécie invasora, distribuição, saúde pública. 


\section{Introdução}

O caramujo exótico invasor Achatina fulica Bowdich, 1822 é nativo do leste da África, sua distribuição original tem limite ao sul em Natal (África do Sul) e ao norte na Somália (Raut \& Barker, 2002). Atualmente, entretanto, devido ao seu sucesso como invasora, sua distribuição abrange quase todos os continentes (África, Américas, Leste e Sul da Ásia e Oceania), inclusive regiões temperadas (e.g., Sul do Japão, Raut \& Barker, 2002).

Atualmente, o Brasil vive a fase explosiva da invasão de A. fulica, estando presente em 24 dos 26 estados brasileiros (sem registro apenas no Acre e Amapá) e no Distrito Federal (Thiengo et al., 2007, AgudoPadrón, 2009). Pelo menos três introduções de A. fulica parecem ter ocorrido no Brasil. Duas invasões foram voluntárias com o objetivo de criação e comercialização dos caramujos (1989 em Curitiba, Paraná e 1996-1998 em Santos, São Paulo) (Teles \& Fontes 2002, Fischer \& Costa, 2010). Enquanto a terceira, com informações menos precisas, parece ter acontecido em 1975, quando um morador de Juiz de Fora, Minas Gerais relatou ter comprado matrizes de A. fulica em uma feira livre (Barçante et al. 2005). Ziller \& Zalba (2007) mencionam ainda uma introdução ocorrida em 1972, mas sem maiores informações.

A. fulica pode transmitir zoonoses, causar danos ao meio ambiente e prejuízos econômicos. Esses danos são geralmente elevados devido ao comportamento gregário e frequentes explosões populacionais da espécie (Mead 1961). Tal infestação aumenta de importância quando consideramos que esse caramujo é hospedeiro intermediário de larvas de nematódeos Metastrogyloidea parasitos do ser humano, bem como de felídeos, cães e outros mamíferos. O nematódeo Angiostrongylus cantonensis (Chen, 1935), agente etiológico da meningite eosinofílica, pode ter $A$. fulica como um dos seus principais hospedeiros (Thiengo et al. 2007). No Brasil, larvas de A. cantonensis foram encontradas em A. fulica procedentes do Estado de São Paulo e casos dessa meningite foram registrados nos Estados do Espírito Santo (Caldeira et al. 2007) e de Pernambuco (Lima et al., 2009). O estudo epidemiológico realizado em Pernambuco mostrou a participação ativa de A. fulica na transmissão (Thiengo et al. 2010), tal como observado em países da Ásia e Ilhas do Pacífico (e.g., Lv et al., 2009).

Já Angiostrongylus costaricensis Morera \& Céspedes, 1971, nematódeo causador de angiostrongilíase abdominal, também está presente em diversos estados brasileiros (RS, SC, PR, MG, ES e SP) além do Distrito Federal e tem diversos moluscos terrestres como hospedeiro intermediário, entre eles A. fulica (Carvalho et al., 2003). Aelurostrongylus abstrusus (Railliet, 1898), nematódeo parasito de pulmão de felídeos causando pneumonia, é o metastrongilídeo mais fequentemente detectado em exemplares de A. fulica examinados no Laboratório de Referência Nacional em Malacologia Médica (LRNM, IOC, FIOCRUZ) (Thiengo et al. 2008). Larvas desse nematódeo foram encontradas em A. fulica provenientes das regiões Nordeste, Centro-Oeste e Sudeste do Brasil.

Exemplares de A. fulica infectados por larvas de nematódeos foram registrados em municípios de diferentes estados do país, incluindo ilhas da costa brasileira, como a Ilha Grande (RJ). Assim, a continuação da explosão populacional descontrolada de A. fulica que ocorre hoje no Brasil pode acarretar o aumento das doenças que tenham essa espécie como transmissor (Graeff-Teixeira 2007). Mundialmente, a ampliação da ocorrência de casos de meningite causada por A. cantonensis parecem estar ligados à dispersão de A. fulica (e.g., Lv et al., 2009). A transmissão dos nematódeos pelo molusco para os demais hospedeiros ocorre através da ingestão de alimento contaminado pelo muco liberado pelo molusco infectado ou do próprio molusco infectado cru ou mal cozido.
A. fulica pode ainda atuar como praga agrícola ou levar a perda da biodiversidade local (Cowie \& Robinson 2003), em razão do seu potencial competitivo, predatório (e.g., Meyer et al., 2008), ou de transmissão de doenças para animais selvagens (e.g., Thiengo et al., 2008). No Brasil, A. fulica põe em risco principalmente espécies de Megalobulimus sp., pois essas são frequentemente confundidas com a espécie invasora e por isso erroneamente sacrificadas. Recentemente, Colley \& Fischer (2009) relataram impactos à biodiversidade causados pela infestação de A. fulica, tanto decorrentes da competição por espaço e alimento com os moluscos nativos, quanto pela interferência na cadeia alimentar.

No Estado do Rio de Janeiro, o número de municípios com registros de ocorrência de A. fulica aumentou sete vezes, de 2002 a 2006, com presença confirmada em pelo menos 57 dos 92 municípios do estado (Thiengo et al., 2007). No presente estudo, apresentamos os novos registros de A. fulica no Estado do Rio de Janeiro com a distribuição atual dessa espécie por municípios, assim como os nematódeos parasitos de importância médica-veterinária associados a essa espécie no estado. Tais dados são fundamentais para eventuais planos de manejo da infestação e ou de erradicação da espécie no território brasileiro.

\section{Material e Métodos}

Novos registros de $A$. fulica foram obtidos principalmente a partir de amostras recebidas no LRNM (IOC/ FIOCRUZ), mas também de publicações da mídia ou oficiais, de consulta às Secretarias Municipais de Saúde, Vigilância Sanitária e Vigilância Ambiental. Os municípios para os quais os órgãos municipais não tinham registro da presença do caramujo foram visitados por nossa equipe. Servidores da vigilância sanitária ou ambiental e representantes da população local foram questionados sobre a presença dessa espécie, apresentando um indivíduo vivo como exemplar. De maneira complementar, busca ativa foi realizada em habitats propícios para essa espécie, como terrenos baldios com entulhos e vegetação. Os exemplares foram identificados pela morfologia externa da concha.

Para a pesquisa de larvas de nematódeos de interesse médicoveterinário, os exemplares vivos de A. fulica coletados ou enviados ao LRNM pelas Secretarias Municipais de Saúde foram digeridos artificialmente através da técnica de Wallace \& Rosen (1969) modificada (Thiengo et al., 2008). As amostras digeridas foram examinadas sob microscópio estereoscópico à procura de larvas de nematódeos.

\section{Resultado e Discussão}

A presença de A. fulica ficou evidenciada em 26 municípios de 35 que Thiengo et al. (2007) considerou livres de invasões à época (Figura 1). Este dado representa um alarmante aumento de, pelo menos, $50 \%$ no número de municípios infestados em apenas quatro anos. Apenas nove (Areal, Bom Jardim, Cambuci, Duas Barras, Engenheiro Paulo de Frontin, Macuco, Rio das Flores, Sumidouro e Varre-Sai) dos 92 municípios do estado ainda não têm registro da presença de A. fulica. Vale a pena ressaltar que esses dados não representam evidências concretas de que a invasão ainda não aconteceu nesses municípios.

Além dos ambientes antrópicos (e.g., quintais, terrenos baldios e cultivos agrícolas), esse caramujo ocorre também em áreas de proteção ambiental, como no Parque Municipal Ecológico Grumari, na Reserva Biológica de Poços das Antas (Faraco \& Lacerda, 2004) e nos Parques Estaduais do Chacrinha, da Pedra Branca (informação do Serviço de planejamento e pesquisa do Estado do Rio de Janeiro) e da Ilha Grande (Santos et al., 2002). No município do Rio de Janeiro, por exemplo, dos 181 bairros existentes, 155 possuem locais de infestação por essa espécie, sendo o primeiro registro dessa espécie para município de 2002 (Thiengo et al., 2007, COMLURB, 2009). 


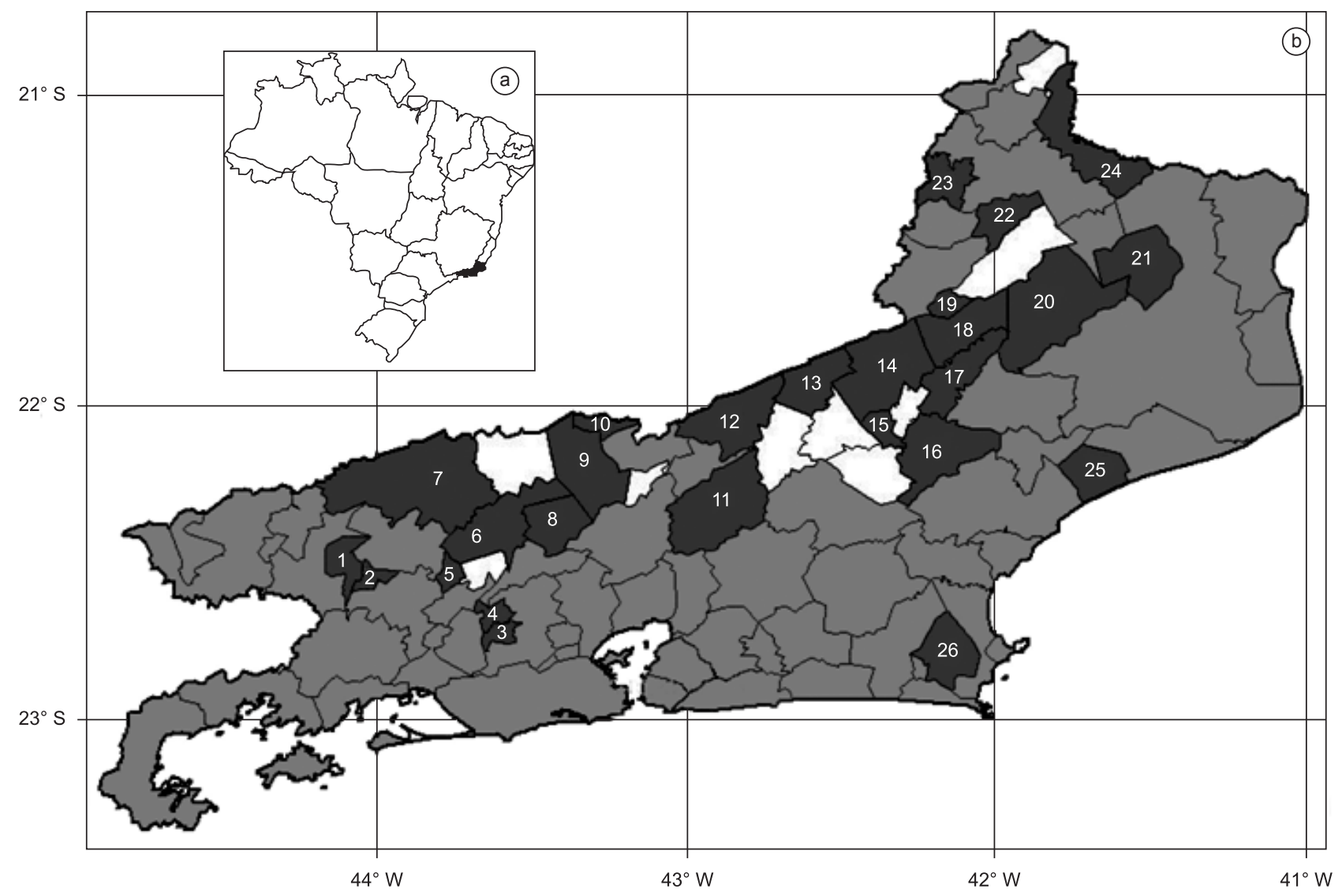

Figura 1. Distribuição de Achatina fulica em municípios do Estado do Rio de Janeiro. a) Mapa do Brasil localizando o Estado do Rio de Janeiro em preto. b) Mapa do Estado do Rio de Janeiro localizando os municípios infestados por A. fulica. Cinza- claro: municípios em que o primeiro registro de $A$. fulica ocorreu até 2006. Cinza- escuro: municípios que representam novas ocorrências de A. fulica. Branco: municípios em que A. fulica ainda não foi encontrada. 1. Volta Redonda, 2. Pinheiral, 3. Queimados, 4. Japeri, 5. Mendes, 6. Vassouras, 7. Valença, 8. Paty do Alferes, 9. Paraíba do Sul, 10. Comendador Levy Gasparian, 11. Teresópolis, 12. Sapucaia, 13. Carmo, 14. Cantagalo, 15. Cordeiro, 16. Trajano de Moraes, 17. São Sebastião do Alto, 18. Itaocara, 19. Aperibé, 20. São Fidélis, 21. Cardoso Moreira, 22. São José de Ubá, 23. Laje do Muriaé, 24. Bom Jesus do Itabapoana, 25. Carapebus, 26. São Pedro da Aldeia.

Figure 1. Distribution of Achatina fulica in municipalities of the State of Rio de Janeiro. a) Map of Brazil highlighting the State of Rio de Janeiro in black. b) Map of the State of Rio de Janeiro showing municipalities infested by A. fulica. Light grey, municipalities in which the presence of $A$. fulica was first registered before 2006. Dark grey, municipalities that represent new occurrences of A. fulica. White, municipalities in which A. fulica has not been found. 1. Volta Redonda, 2. Pinheiral, 3. Queimados, 4. Japeri, 5. Mendes, 6. Vassouras, 7. Valença, 8. Paty do Alferes, 9. Paraíba do Sul, 10. Comendador Levy Gasparian, 11. Teresópolis, 12. Sapucaia, 13. Carmo, 14. Cantagalo, 15. Cordeiro, 16. Trajano de Moraes, 17. São Sebastião do Alto, 18. Itaocara, 19. Aperibé, 20. São Fidélis, 21. Cardoso Moreira, 22. São José de Ubá, 23. Laje do Muriaé, 24. Bom Jesus do Itabapoana, 25. Carapebus, 26. São Pedro da Aldeia.

O movimento de indivíduos de A. fulica varia ao longo do ciclo de vida (Tomiyama \& Nakane 1993) e estações do ano (Raut \& Barker 2002). Exemplares jovens em geral são dispersores mais eficientes que os adultos (Tomiyama \& Nakane 1993). Porém, a maior parte da dispersão dessa espécie é mediada pelo ser humano de maneira voluntária ou involuntária (e.g., através de cargas infestadas; Wolfenbarger 1971). Tal forma de dispersão passiva de A. fulica possivelmente reflete sua expansão e ocorrência no Estado do Rio de Janeiro, possuindo municípios sem registros do invasor limítrofes às áreas infestadas (Figura 1). Indivíduos jovens são facilmente transportados involuntariamente devido ao seu pequeno tamanho e o desconhecimento da população de que esses indivíduos pequenos se tornam adultos grandes que causam problemas sanitários. O estabelecimento e a proliferação de $A$. fulica ocorrem de forma mais eficiente em ambientes com sítios de repouso (e.g., entulho de obra, cercas vivas) e alimentação (e.g., restos de lixo, plantas ornamentais) abundantes (Fischer \& Colley 2005).
Colley (2010), após avaliar as medidas de controle de A. fulica existentes (controle biológico, químico e físico), relatou que a primeira etapa do manejo é diagnosticar o perfil de ocupação do invasor, para escolher posteriormente a melhor medida a ser utilizada e adaptá-la a realidade local. No município do Rio de Janeiro, a Companhia Municipal de Limpeza Urbana (COMLURB) junto com a Defesa Civil do município lançaram uma força tarefa a fim de controlar as populações de A. fulica e conscientizar a população local para que também atue no controle. Essa força tarefa conta com pessoal exclusivo, que utiliza documentos para notificação e educação, e simultaneamente realiza coleta ativa e incineração dos indivíduos no controle (COMLURB, 2009). Outro exemplo é o município de Macaé, que no seu programa de controle de A. fulica também utiliza uma equipe de funcionários da Prefeitura e a população local. As atividades, realizadas desde 2006, incluem mutirão de coleta ativa e palestras de conscientização em escolas públicas e privadas (Barboza, 
2009) e informação sobre o controle e os cuidados no manuseio de A. fulica no "Guia de Proteção do Cidadão" (Secretaria Executiva de Defesa Civil 2009). Alguns municípios têm feito uso de moluscicidas (e.g., produtos a base de metaldeído) no controle desse caramujo. Porém, tais produtos não são recomendados pela sua toxicidade e por não serem específicos para A. fulica, matando também outras espécies de moluscos. Além disso, tais produtos são danosos a outros organismos, como pássaros e outros predadores que se alimentam do veneno ou do caramujo envenenado (Barker \& Watts 2002).

A pesquisa da helmintofauna presente em A. fulica obtida no Estado do Rio de Janeiro, até 2007 (Thiengo et al., 2008), resultou no registro de A. abstrusus e/ou Strongyluris-like em sete municípios (Campos dos Goytacazes, Duque de Caxias, Mangaratiba, Maricá, Niterói, Rio de Janeiro e São Gonçalo). O prosseguimento desta pesquisa com o exame de 1.102 indivíduos adicionais de $A$. fulica amplia a ocorrência destas formas larvais para outros quatro municípios (Angra dos Reis, Barra do Piraí, Bom Jesus de Itabapoana e Valença) e registra a presença de Rhabditis sp. em A. fulica procedentes de Bom Jesus de Itabapoana, Barra do Piraí, Duque de Caxias, Niterói, Rio de Janeiro, São Gonçalo e Valença. Algumas das amostras obtidas em Angra dos Reis, Barra do Piraí, Bom Jesus de Itabapoana, Duque de Caxias, Maricá, Niterói, Rio de Janeiro, São Gonçalo, São João de Meriti, São Sebastião do Alto e Valença apresentam ainda outras formas larvais que requerem estudos complementares visando a identificação específica do nematódeo. Estes registros corroboram estudos anteriores (e.g., Thiengo \& Fernandez, 2010) de que $A$. fulica já se encontra inserida em ciclos de vida de diversos parasitos da fauna silvestre e doméstica no Brasil.

A rápida ampliação da distribuição de A. fulica no Estado do Rio de Janeiro chama mais uma vez atenção para a importância de planos de manejo e controle dessa espécie. É importante que esses planos sejam contínuos e generalizados em todos os municípios uma vez que a re-infestação ocorre de maneira acelerada (e.g., Simião \& Fischer 2004). Municípios que ainda não registraram a presença de A. fulica deveriam investir em medidas preventivas, como campanhas de conscientização para que a população local não transporte esse caramujo e seja capaz de detectar a introdução deste molusco em sua fase inicial, facilitando consideravelmente o controle da invasão. Considerando o nível atual de infestação do estado, a erradicação de A. fulica é pouco provável, requerendo um grande investimento econômico. Entretanto, medidas de controle que mantenham as populações de $A$. fulica em níveis toleráveis são factíveis, além de extremamente necessárias.

\section{Agradecimentos}

Os autores são gratos aos funcionários das Secretarias Municipais de Saúde, Vigilância Sanitária e Vigilância Ambiental pela atenção e informações providas; e aos biólogos Pablo Menezes Coelho e Daniel Viggiano Lago (LRNM, IOC, FIOCRUZ) pelo valioso auxílio nos trabalhos de campo. Financiamento CNPq (processo 518181/2008-0) e FAPERJ (processos CNE 102.680/2008 e E-26/102.007/2009).

\section{Referências Bibliográficas}

AGUDO-PADRÓN, I.A. 2009. Recent terrestrial and freshwater molluscs of Rio Grande do Sul State, RS, Southern Brazil Region: a comprehensive synthesis and check list. Visaya Agosto: 2-14.

BARBOZA, L. http://www.macae.rj.gov.br/noticias/mostranot.asp?id=16851 (último acesso em 15/01/2010)

BARÇANTE, J.M.P., BARÇANTE, T.A., DIAS, S.R.C. \& LIMA, W.S. 2005. Ocorrência de Achatina fulica Bowdich, 1822 (Mollusca: Gastropoda: Achatinoidea) no Estado de Minas Gerais, Brasil. Bol. Mus. Biol. Mello Leitao 18:65-70.
BARKER, G.M. \& WATTS, C. 2002. Management of the invasive alien snail Cantareus aspersus on conservation land. Department of Conservation, Wellington.

BOWDICH, T.E. 1822. Elements of conchology, including the fossil genera and the animals, 1. Univalves. Smith, Paris.

CALDEIRA, R.L., MENDONÇA, C.L., GOVEIA, C.O., LENZI, H.L., GRAEFF-TEIXEIRA, C., LIMA, W.S., MOTA, E.M., PECORA, I.L., MEDEIROS, A.M.Z. \& CARVALHO, O.S. 2007. First record of molluscs naturally infected with Angiostrongylus cantonensis (Chen, 1935) (Nematoda: Metastrongylidae) in Brazil. Mem. Inst. Oswaldo Cruz 102(7):887-889.

CARVAlho, O.S., TEles, H.M.S., MOTA, E.M., LAFETÁ, C., MENDONÇA, G.F. \& LENZI, H.L. 2003. Potentiality of Achatina fulica Bowdich, 1822 (Mollusca: Gastropoda) as intermediate host of the Angiostrongylus costaricensis Morera \& Céspedes 1971. Rev. Soc. Bras. Med. Trop. 36(6):743-745.

CHEN, H.T. 1935. Un nouveau nematode pulmoaire: Pulmonema cantonensis n. g. n. sp., des rats de Canton. Ann. Parasit. Hum. Comp. 13:312-317.

COLLEY, E. \& FISCHER, M.L. 2009. Avaliação dos problemas enfrentados no manejo do caramujo gigante africano Achatina fulica (Gastropoda: Pulmonata) no Brasil. Zoologia 26(4):674-683.

COLLEY, E. 2010. Medidas de controle de Achatina fulica. In O caramujo gigante africano Achatina fulica no Brasil (M.L. Fischer \& L.C.M. Costa, eds). Champagnat Editora - PUCPR, Curitiba, p. 203-229.

COMLURB. Vetores. http://comlurb.rio.rj.gov.br/serv_vetores.htm (último acesso em 15/01/2010)

COWIE, R.H. \& ROBINSON, D.G. 2003. Pathways of introduction of nonindigenous land and freshwater snails and slugs. In Invasive species: vectors and management strategies (G. Ruiz \& J.T. Carlton, ed.). Island Press, Washington, D.C., p. 93-122.

FARACO, F.A. \& LACERDA, A.C.R. 2004. Contaminação biológica em unidades de conservação - o caso do caramujo africano (Achatina fulica, Mollusca, Gastropoda). In Congresso Brasileiro de Unidades de Conservação. Fundação O Boticário de Proteção à Natureza, Rede Nacional Pró Unidades de Conservação, Curitiba, p. 78-84.

FISCHER, M.L. \& COLLEY, E. 2005. Espécie invasora em reservas naturais: caracterização de população de Achatina fulica Bowdich, 1822 (Mollusca - Achatinidae) na Ilha Rasa, Guaraqueçaba, Paraná, Brasil. Biota Neotrop. 5(1):1-18. http://www.biotaneotropica.org.br/v5n1/pt/ abstract?article+BN03305012005 (último acesso em 15/01/2010)

FISCHER, M.L. \& COSTA, L.C.M. 2010. O caramujo gigante africano Achatina fulica no Brasil. Champagnat Editora - PUCPR, Curitiba, 269p.

GRAEFF-TEIXEIRA, C. 2007. Expansion of Achatina fulica in Brazil and potential increased risk for angiostrongyliasis. Trans. Roy. Soc. Trop. Med. Hyg. 101:743-744.

LIMA, A.R.M.C., MESQUITA, S.D., SANTOS, S.S., AQUINO, E.R.P.D., ROSA, L.R.S., DUARTE, F.S., TEIXEIRA, A.O., COSTA, Z.R.S. \& FERREIRA, M.L.B. 2009. Alicata disease: neuroinfestation by Angiostrongylus cantonensis in Recife, Pernambuco, Brazil. Arq. NeuroPsiquiatr. 67(4): 1093-1096.

LV, S., ZHANG, Y., LIU, H.-X., HU, L., YANG, K., STEINMANN, P., CHEN, Z., WANG, L.-Y., UTZINGER, J. \& ZHOU, X.-N. 2009. Invasive snails and an emerging infectious disease: results from the first national survey on Angiostrongylus cantonensis in China. PLoS Negl. Trop. Dis. 3(2):e368. doi:310.1371/journal.pntd.0000368.

MEAD, A.R. 1961. The giant African snail: a problem in economic malacology. The University of Chicago Press, Chicago.

MEYER, W.M., HAYES, K.A. \& MEYER, A.L. 2008. Giant African snail, Achatina fulica, as a snail predator. Am. Malacol. Bull. 24:117-119.

MORERA, P. \& CÉSPEDES, R. 1971. Angiostrongilosis abdominal. Una nueva parasitosis humana. Acta Med. Costarric. 14:159-173.

RAILLIET, A. 1898. Rectification de la nomenclature d'après les traveaux récents. Recl. Med. Vet. 75:171-174. 
RAUT, S.K. \& BARKER, G.M. 2002. Achatina fulica Bowdich and other Achatinidae as pests in tropical agriculture. In Molluscs as crop pests (G.M. Barker, ed.). CABI Publishing, Wallingford, p. 55-114.

SANTOS, S.B., MONTEIRO, D.P. \& THIENGO, S.C. 2002. Achatina fulica (Mollusca, Achatinidae) na Ilha Grande, Angra dos Reis, Rio de Janeiro: implicações para a saúde ambiental. Biociências 10(2):159-162.

SECRETARIA EXECUTIVA DE DEFESA CIVIL. 2009. Guia de proteção do cidadão. 2 ed. Secretaria Executiva de Defesa Civil, Macaé.

SIMIÃO, M.S. \& FISCHER, M.L. 2004. Estimativa e inferências do método de controle do molusco exótico Achatina fulica Bowdich 1822 (Stilommatophora; Achatinidae) em Pontal do Paraná, Litoral do Estado do Paraná. Cad. Biodivers. 4(2):74-83.

TELES, H.M.S. \& FONTES, L.R. 2002. Implicações da introducção e dispersão de Achatina fulica Bowdich, 1822 no Brasil. Bol. Inst. Adolfo Lutz 12:3-5.

THIENGO, S.C. \& FERNANDEZ, M.A. 2010. Achatina fulica: um problema de saúde pública? In O caramujo gigante africano Achatina fulica no Brasil (M.L. Fischer \& L.C.M. Costa, eds). Champagnat Editora - PUCPR, Curitiba, p. 189-202.

THIENGO, S.C., FARACO, F.A., SALGADO, N.C., COWIE, R.H. \& FERNANDEZ, M.A. 2007. Rapid spread of an invasive snail in South
America: the giant African snail, Achatina fulica, in Brasil. Biol. Invasions 9:693-702.

THIENGO, S.C., FERNANDEZ, M.A., TORRES, E.J.L., COELHO, P.M. \& LANFREDI, R.M. 2008. First record of a nematode Metastrongyloidea (Aelurostrongylus abstrusus larvae) in Achatina (Lissachatina) fulica (Mollusca, Achatinidae) in Brazil. J. Invertebr. Pathol. 98:34-39.

THIENGO, S.C., MALDONADO, A., MOTA, E.M., TORRES, E.J.L., CALDEIRA, R., CARVALHO, O.S., OLIVEIRA, A.P.M., SIMÕES, R.O., FERNANDEZ, M.A. \& LANFREDI, R.M. in press. The giant African snail Achatina fulica as natural intermediate host of Angiostrongylus cantonensis in Pernambuco, Northeast Brazil. Acta Tropica.

TOMIYAMA, K. \& NAKANE, M. 1993. Dispersal patterns of the giant African snail, Achatian fulica (Férussac) (Stylommatophora: Achatinidae), equipped with a radio-transmitter. J. Moll. Stud. 59:315-322.

WALLACE, G.D. \& ROSEN, L. 1969. Studies on eosinophilic meningitis. V. Molluscan hosts of Angiostrongylus cantonensis on the Pacific Islands. Am. J. Trop. Med. Hyg. 18:206-216.

WOLFENBARGER, D.O. 1971. Dispersion of the giant African snail Achatina fulica. Quart. Jour. Florida Acad. Sci. 34(1):48-52.

ZILLER, S.R. \& ZALBA, S. 2007. Propostas de ação para prevenção e controle de espécies exóticas invasoras. Nat. Conserv. 5(2):8-15. 